PROCEEDINGS OF THE

AMERICAN MATHEMATICAL SOCIETY

Volume 99, Number 2, February 1987

\title{
ON THE FREE GENUS OF KNOTS
}

\author{
YOAV MORIAH
}

\begin{abstract}
The class of knots consisting of twisted Whitehead doubles can have arbitrarily large free genus but all have genus 1 .
\end{abstract}

1. Introduction. Let $K \subset S^{3}$ be a knot. The free genus of $K$ denoted by $g_{f}(K)$ is the minimal genus of all Seifert surfaces $S$ for $K$ such that $\pi_{1}\left(S^{3}-\stackrel{\circ}{N}(S)\right)$ is free. Let $g(K)$ denote the genus of $K$. Note that $g_{f}(K) \geq g(K)$ for all knots. It is conjectured (see [1, Problem 1.20a]) that the difference between the free genus and the genus can be arbitrarily large. In this paper we show that the conjecture is true by considering twisted Whitehead doubles. ${ }^{1}$ The conjecture will follow from the following theorem.

THEOREM 1. Let $D_{k}(K), k \neq 0$, denote the Whitehead double with $k$ twists of a knot $K \subset S^{3}$. Suppose that

$$
\operatorname{rank} H_{1}\left(B_{|4 k+1|}(K)\right)=r
$$

where $\operatorname{rank}(G)$ is the minimal number of generators for $G$ and $B_{n}(K)$ is the $n$-fold cyclic cover of $S^{3}$ branched over $K$. Then

$$
g_{f}\left(D_{k}(K)\right) \geq \frac{2 r-1}{2|4 k+1|} .
$$

Note. $g\left(D_{k}(K)\right)=1$ for all knots $K$ and all $k \in \mathbf{Z}$. The conjecture follows from the theorem by the following example:

Let $K^{n}$ be the connected sum of $n$ trefoils and let $k=-1$. Then $|4 k+1|=3$ and rank $H_{1}\left(B_{3}\left(K^{n}\right)\right)=2 n$. From the theorem it follows that $g_{f}\left(D_{-1}\left(K^{n}\right)\right) \geq$ $(4 n-1) / 6$ while $g\left(D_{-1}\left(K^{n}\right)\right)=1$. Let $n \rightarrow \infty$ and the conjecture follows.

I would like to thank Professor C. Mc A. Gordon for many useful conversations concerning this work.

2. Terminology and preliminary lemmas. Let $K \subset S^{3}$ be an unoriented knot in $S^{3} . N(K)$ will denote a tubular neighborhood of $K$. The exterior of $K$ is $X=S^{3}-\stackrel{\circ}{N}(K)$. Let $f:\left(S^{1} \times D^{2}\right) \rightarrow N(K)$ be the 0 framing homeomorphism that is $\left[f\left(S^{1} \times *\right)\right]=0 \in H_{1}(X)$ for $* \in \partial D^{2}$. Choosing an orientation for $K$ determines a longitude meridian pair $\lambda, \mu \in H_{1}(\partial X)$ as follows:

$$
\lambda=\left[f\left(S^{1} \times *\right)\right] \quad \text { where } * \in \partial D^{2} \text { and } \mu=\left[f\left(* \times \partial D^{2}\right)\right], \quad * \in S^{1} .
$$

Let $r=m / n$ where $m, n \in \mathbf{Z}, n=0$ is allowed.

Received by the editors January 10, 1985 .

1980 Mathematics Subject Classification (1985 Revision). Primary 57M25.

${ }^{1}$ It is still unknown for untwisted doubles as suggested in [1, Problem 1.20a].

(C)1987 American Mathematical Society $0002-9939 / 87 \$ 1.00+\$ .25$ per page 


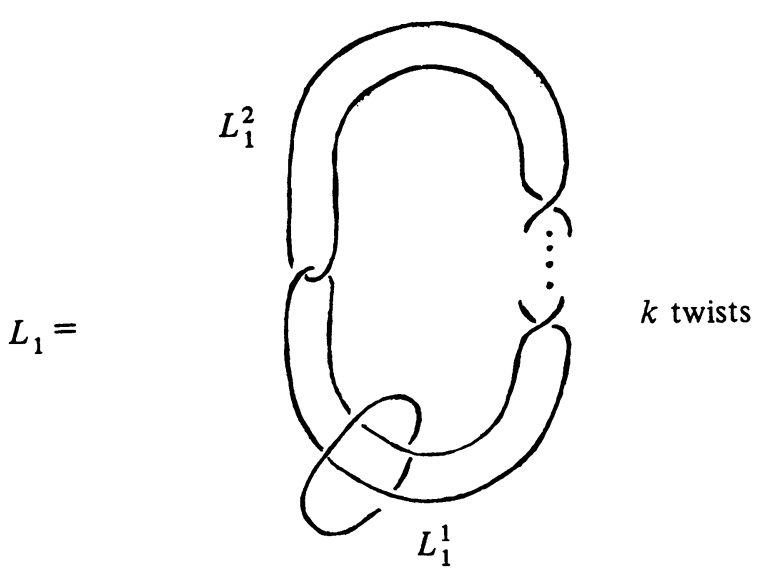

FIGURE 1

The manifold obtained by doing Dehn surgery of type $r$ on $K$ is $X \cup_{h} S^{1} \times D^{2}$ where $h: S^{1} \times \partial D^{2} \rightarrow \partial X$ is a homeomorphism such that $\left[h\left(* \times \partial D^{2}\right)\right]=m \mu+n \lambda \in$ $H_{1}(\partial X)$.

We need the following three lemmas.

LEMMA 1. Let $K \subset S^{3}$ be a knot. Let $X=S^{3}-\stackrel{\circ}{N}(K)$ and let $g_{f}(K)$ denote the minimal genus of all unknotted Seifert surfaces for $K$. Let $\operatorname{rank} \pi_{1}(X)$ denote the minimal number of generators for $\pi_{1}(X)$. Then

$$
\operatorname{rank} \pi_{1}(X) \leq g_{f}(K)+1 .
$$

LEMMA 2. Let $G$ be a finitely generated group and $H \subset G$ be a subgroup of finite index $k$ in $G$. Then

$$
\operatorname{rank}(G) \geq \frac{\operatorname{rank}(H)-1}{k}+1
$$

The proofs of the above lemmas will appear in $\S 4$.

LEMMA 3. $H_{1}\left(B_{2}\left(D_{k}(K)\right)\right) \cong \mathbf{Z}_{|4 k+1|}$.

REMARK. This can be proved easily by computing the Seifert matrices for $D_{k}(K)$. We will prove it via the following construction which is needed for the main theorem.

PROOF. Fix an orientation for $K$. Now we have a well-defined longitude meridian pair $\lambda, \mu$ in $H_{1}(\partial X)=H_{1}\left(\partial\left(S^{3}-\stackrel{\circ}{N}(K)\right)\right)$. We can think of $S^{3}-\stackrel{\circ}{N}\left(D_{k}(K)\right)$ as the complement in $S^{3}$ of the link $L_{1}$ with $X=S^{3}-\stackrel{\circ}{N}(K)$ glued to the boundary component corresponding to $L_{1}^{1}$ (see Figure 1).

The gluing map $h$ for $\partial X$ is given by

$$
\mu_{x} \stackrel{h_{*}}{\rightarrow} 0 \cdot \mu_{L_{1}^{1}}+1 \cdot \lambda_{L_{1}^{1}}, \quad \lambda_{x} \stackrel{h_{*}}{\rightarrow} 1 \cdot \mu_{L_{1}^{1}}+0 \cdot \lambda_{L_{1}^{1}}
$$

or in the matrix formed by $h_{*}=\left[\begin{array}{ll}0 & 1 \\ 1 & 0\end{array}\right]$. We can twist about $L_{1}^{1}-k$ times and use the symmetry of the Whitehead link to get the following description of $S^{3}-\stackrel{\circ}{N}\left(D_{k}(K)\right)$ 

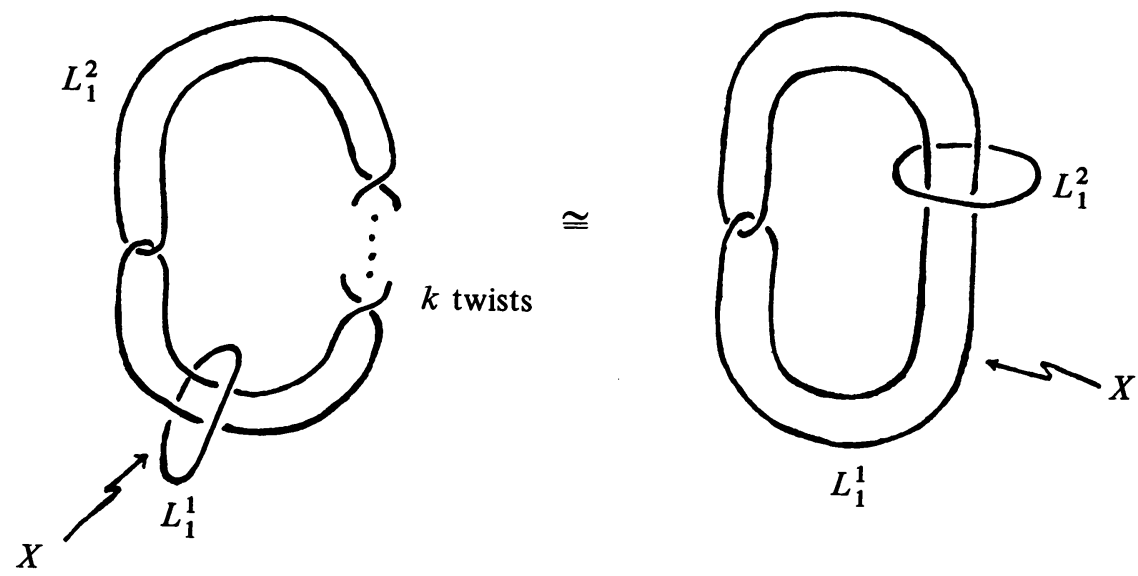

FIGURE 2

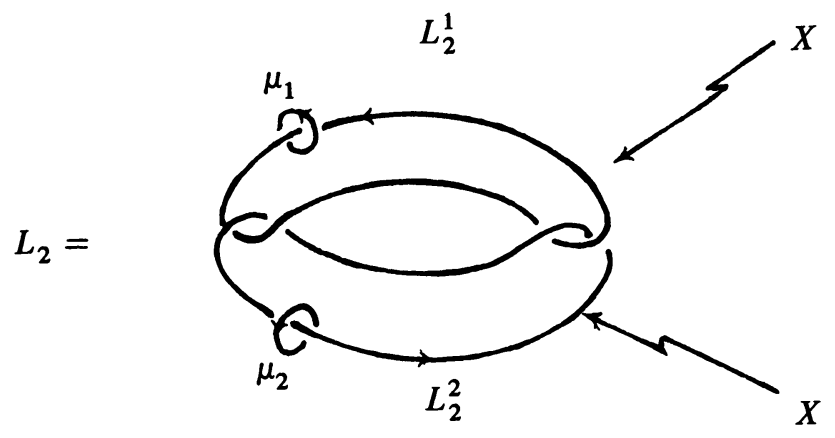

FIGURE 3

(see Figure 2) where the surgery instructions for $\partial X$ are given by the map

$$
h_{*}=\left(\begin{array}{cc}
1 & 0 \\
-k & 1
\end{array}\right)\left(\begin{array}{ll}
0 & 1 \\
1 & 0
\end{array}\right)
$$

(see [2, pp. 264-267]).

Let $i$ be the inclusion map $i: X \rightarrow S^{3}-\stackrel{\circ}{N}\left(D_{k}(K)\right)$. A meridian of $X$ is mapped by $i$ to a longitude of $L_{1}^{1}$ which is null homologous in the complement of $D_{k}(K)$. Since $\pi_{1}(X)$ is generated by meridians, it follows that $X$ lifts to the branched double cover of $S^{3}$ branched along $D_{k}(K)$. In other words $B_{2}\left(D_{k}(K)\right)$ can be thought of as the 3-manifold obtained by gluing two copies of $X=S^{3}-\stackrel{\circ}{N}(K)$, one to each boundary component, of the complement in $S^{3}$ of the above link $L_{2}$ (see Figure 3 ).

In terms of the obvious longitude meridian pair $\lambda_{i} \mu_{i}, i=1,2$ of each unknotted components $L_{2}^{1}$ and $L_{2}^{2}$ of $L_{2}$, a longitude of $L_{1}^{1}$ lifts to $1 \cdot \lambda_{i}-2 \cdot \mu_{i}$. Hence, the gluing map for $\partial X$ is given by the matrix

$$
h_{*}=\left[\begin{array}{cc}
2 k+1 & -2 \\
-k & 1
\end{array}\right]\left[\begin{array}{ll}
0 & 1 \\
1 & 0
\end{array}\right] \text {. }
$$


Note that $H_{1}\left(S^{3}-\stackrel{\circ}{N}\left(L_{2}\right)\right) \cong \mathbf{Z}_{\mu_{1}} \oplus \mathbf{Z}_{\mu_{2}}$. A longitude of the unknotted component $L_{2}^{1}$ is homologous to $2 \mu_{2}$. Therefore, because of the symmetry between $L_{2}^{1}, L_{2}^{2}$ gluing the two copies of $X$ gives us the following relations:

$$
(2 k+1) \mu_{1}-2 k \mu_{2}=0, \quad-2 k \mu_{1}+(2 k+1) \mu_{2}=0 .
$$

The relation matrix

$$
\left(\begin{array}{cc}
2 k+1 & -2 k \\
-2 k & 2 k+1
\end{array}\right)
$$

is equivalent to the matrix $(4 k+1)$ so $H_{1}\left(B_{2}\left(D_{k}(K)\right)\right)$ is a cyclic group of order $|4 k+1|$ generated by $\bar{\mu}_{1}$, the image of $\mu_{1}$ in $\mathbf{Z}_{|4 k+1|}$.

3. Proof of the main theorem. Let $W_{n}\left(D_{k}(K)\right)$ denote the $n$-fold cyclic cover of $S^{3}-\stackrel{\circ}{N}\left(D_{k}(K)\right)$. Recall that $H_{1}\left(W_{n}\right)=H_{1}\left(B_{n}\left(D_{k}(K)\right)\right) \oplus \mathbf{Z}$. By Lemma 3 we have a map $\varphi: \pi_{1}\left(W_{2}\left(D_{k}(K)\right)\right) \rightarrow \mathbf{Z}_{|4 k+1|}$. Let $W$ denote the $|4 k+1|$ cyclic cover of $W_{2}\left(D_{k}(K)\right)$ corresponding to $\varphi$, hence $\pi_{1}(W)$ is a subgroup of index $2 \cdot|4 k+1|$ of $\pi_{1}\left(S^{3}-D_{k}(K)\right)$. By Lemma 2

$$
\operatorname{rank} \pi_{1}\left(S^{3}-D_{k}(K)\right) \geq \frac{\operatorname{rank} \pi_{1}(W)-1}{2 \cdot|4 k+1|}+1 .
$$

Let $Y$ denote the $|4 k+1|$ cyclic cover of $B_{2}\left(D_{k}(K)\right)$. Note that $\operatorname{rank} \pi_{1}(W) \geq$ $\operatorname{rank} H_{1}(W) \geq \operatorname{rank} H_{1}(Y)$. Suppose that rank $H_{1}(Y)=2 r$; then by Lemma 1

$$
g_{f}\left(D_{k}(K)\right) \geq \operatorname{rank} \pi_{1}\left(S^{3}-D_{k}(K)\right)-1 \geq \frac{2 r-1}{2 \cdot|4 k+1|} .
$$

Therefore in order to complete the proof it is sufficient to show that if

$$
\operatorname{rank} H_{1}\left(B_{|4 k+1|}(K)\right)=r
$$

then $\operatorname{rank} H_{1}(Y)=2 r$.

REMARK. If we choose to consider $\operatorname{rank} H_{1}(W)$ instead of $\operatorname{rank} H_{1}(Y)$ we can obtain a slightly better formula, i.e.,

$$
g_{f}\left(D_{k}(K)\right) \geq \frac{2 r-1+|4 k+1|}{2 \cdot|4 k+1|} .
$$

We omit the details.

From the proof of Lemma 3, we see that the image of a meridian $\mu$ of $X$ under the map $i_{*}$ induced by the inclusion $i: X \hookrightarrow B_{2}\left(D_{k}(K)\right)$ is $2 \bar{\mu}_{1}$. Since $(4 k+1,2)=1, \mu$ is mapped onto a generator of $H_{1}\left(B_{2}\left(D_{k}(K)\right)\right)$. If $i_{*}(n \mu)=0$ then $n=(4 k+1) m$ and $i_{*}(|4 k+1| \mu)=0$. Hence the $|4 k+1|$ cyclic cover of $X$ lifts to $Y$ and in $Y$ we have exactly two copies of the $|4 k+1|$ cyclic cover of $X$.

Following [1, pp. 264-267] we have description for $B_{2}\left(D_{k}(K)\right)$ as in Figure $4\left(r_{0}\right.$ will denote the surgery instructions for $L^{0}$ ).

The twist homeomorphism about $L^{0}$ changes the gluing map $h$ for $X$ to

$$
h=\left(\begin{array}{cc}
1 & 0 \\
-k & 1
\end{array}\right)\left(\begin{array}{ll}
0 & 1 \\
1 & 0
\end{array}\right) \text {. }
$$




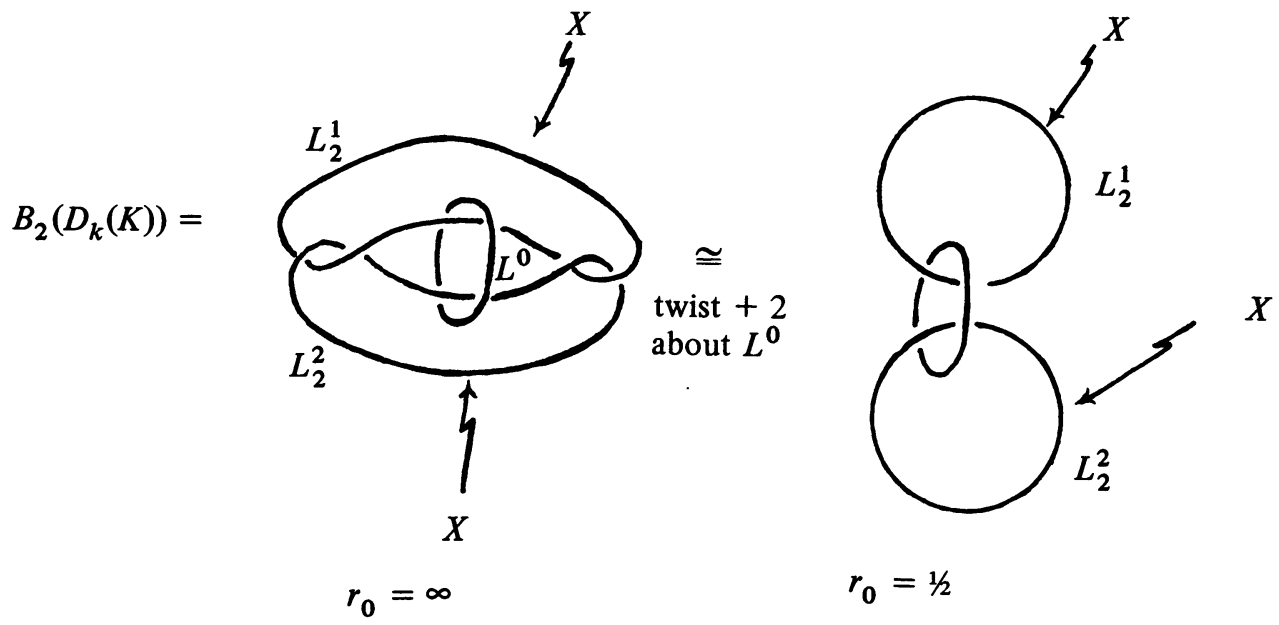

Figure 4

$B_{2}\left(D_{k}(K)\right)=$

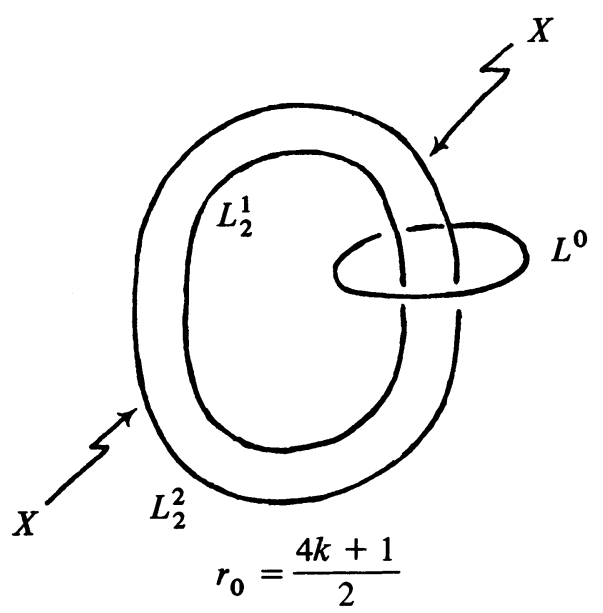

FIGURE 5

Now twist $+k$ times about $L_{2}^{1}$ and $+k$ times about $L_{2}^{2}$ to get Figure 5 with gluing map $h$ given by

$$
\left(\begin{array}{ll}
1 & 0 \\
0 & 1
\end{array}\right)\left(\begin{array}{ll}
0 & 1 \\
1 & 0
\end{array}\right)=\left(\begin{array}{ll}
0 & 1 \\
1 & 0
\end{array}\right)
$$

That is $B_{2}\left(D_{k}(K)\right)$ is the lens space $L_{(|4 k+1|, 2)}$ with two solid tori removed and two copies of $X$ glued in. Let $\tilde{X}_{|4 k+1|}$ denote the $|4 k+1|$ cyclic cover of $X$. The $|4 k+1|$ cyclic cover of $\left(L_{(|4 k+1|, 2)}-\stackrel{\circ}{N}\left(L_{2}\right)\right)$ is $S^{3}-2$ tori. Hence $Y$ is the complement in $S^{3}$ of the two-component link $\tilde{L}$ (see Figure 6) with two copies of $\tilde{X}_{|4 k+1|}$ glued to the boundary components of $\tilde{L}$.

In terms of the obvious longitude meridian pair for each unknotted component $\tilde{L}_{i}$ the gluing map $h$ is given by $h=\left(\begin{array}{ll}0 & 1 \\ 1 & 0\end{array}\right)$. Note that the surgery coefficient $r_{0}=|4 k+1| / 2$ has lifted to $\tilde{r}_{0}=1 / 2$. 
$\tilde{L}=$

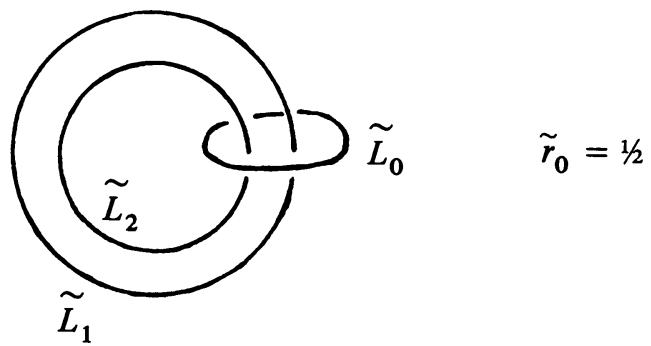

FIGURE 6

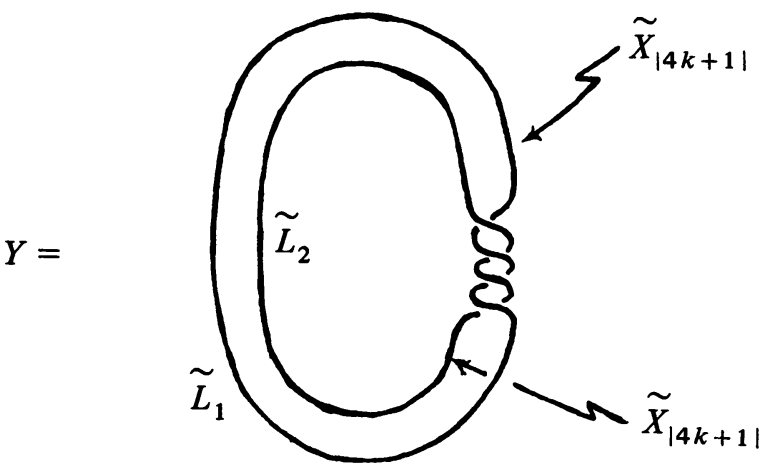

FIGURE 7

After twisting -2 times about $\tilde{L}_{0}$ we get Figure 7 and

$$
h=\left(\begin{array}{cc}
1 & -2 \\
0 & 1
\end{array}\right)\left(\begin{array}{ll}
0 & 1 \\
1 & 0
\end{array}\right) .
$$

Recall that $H_{1}\left(\tilde{X}_{|4 k+1|}\right)=\mathbf{Z} \oplus H_{1}\left(B_{|4 k+1|}(K)\right)$ where $\mathbf{Z}$ is generated by $\tilde{\mu}$ the lift of a meridian $\mu$ of $X$.

$h$ maps $\tilde{\mu}$ to a $-2 / 1$ curve on the boundary components of $S^{3}-\tilde{L}$. The $-2 / 1$ curve is null homologous in $S^{3}-\tilde{L}$ and it follows by the Mayer Vietoris sequence that

$H_{1}(Y)=H_{1}\left(S^{3}-\tilde{L} \cup_{h_{1}} \tilde{X}_{|4 k+1|} \cup_{h_{2}} \tilde{X}_{|4 k+1|}\right) \cong H_{1}\left(B_{|4 k+1|}(K)\right) \oplus H_{1}\left(B_{|4 k+1|}(K)\right)$ by the assumption $\operatorname{rank} H_{1}\left(B_{|4 k+1|}(K)\right)=r$. So $\operatorname{rank}\left(H_{1}(Y)\right)=2 r$ and the proof is complete.

Let $K$ be a knot in $S^{3}$ and let $t(K)$ denote the tunnel number of $K$, that is the minimal number of 2 handles that need be removed from $S^{3}-\stackrel{\circ}{N}(K)$ so that the complement is a handlebody. $g(K)$ as before denotes the genus of $K$.

COROLlARY. $t(K)-g(K)$ can be arbitrarily large.

PROOF. $\pi_{1}\left(S^{3}-K\right)$ has a presentation with $t(K)+1$ generators and $t(K)$ relators so $\operatorname{rank} \pi_{1}\left(S^{3}-K\right) \leq t(K)+1$. Let $K^{n}$ be the connected sum of $n$ trefoils and set $k=-1, g\left(D_{-1}\left(K^{n}\right)\right)=1$ and $\operatorname{rank} \pi_{1}\left(S^{3}-D_{-1}\left(K^{n}\right)\right) \geq(4 n-1) / 6$ so $t\left(D_{-1}\left(K^{n}\right)\right) \geq(4 n-7) / 6$. Now set $n$ arbitrarily large. 


\section{Proof of lemmas.}

ProOF OF LEMMA 1 . Let $S$ be a Seifert surface for $K$ which realizes the free genus. Let $\stackrel{\circ}{N}(S)$ be a product neighborhood of $S$ in $S^{3}-\stackrel{\circ}{N}(K) . S^{3}-\stackrel{\circ}{N}(S)$ is a handlebody of genus $g_{f}(K)$. Hence $\pi_{1}\left(S^{3}-\stackrel{\circ}{N}(K)\right)$ is an HNN extension of the free group with $g_{f}(K)$ generators and has a presentation with $g_{f}(K)+1$ generators $\left[3\right.$, p. 180]. So $\operatorname{rank} \pi_{1}\left(S^{3}-\stackrel{\circ}{N}(K)\right) \leq g_{f}(K)+1$.

PROOF OF LEMMA 2. Let $\left\langle X_{1}, \ldots, X_{g} \mid R_{1}, \ldots, R_{l}\right\rangle$ be a presentation for $G$ with $g$ minimal. Construct a 2 complex $K$ with 1 vertex $g$ edges and $l$ faces such that $\pi_{1}(K)=G$. $K$ has a $k$ fold cover $\tilde{K}$ such that $\pi_{1}(\tilde{K})=H . \tilde{K}$ has $k$ vertices, $k \cdot g$ edges and $k \cdot l$ faces. Shrink a maximal tree in the 1 skeleton of $\tilde{K}$.

Set a base point for $\pi_{1}(\tilde{K})$ at the remaining vertex. We have a 2 complex with 1 vertex, $k g-k+1$ edges and $k l$ faces. This gives rise to a presentation for $H$ with $k g-k+1$ generators. If $h$ is the minimal number of generators in any presentation for $H$ then

$$
k g-k+1 \geq h \quad \text { so } \quad g \geq(h-1) / k+1
$$

\section{REFERENCES}

1. R. Kirby, Problems in low dimensional manifold theory, Proc. Sympos. Pure Math., vol. 32, part 2, Amer. Math. Soc., Providence, R.I., 1978, pp. 273-312.

2. D. Rolfsen, Knots and links, Publish or Perish, Boston, Mass., 1976.

3. R. Lyndon and P. Schupp, Combinatorial group theory, Springer-Verlag, 1977. 78712

Department of Mathematics, University of TeXas at Austin, Austin, TeXas

Current address: Department of Mathematics, University of British Columbia, Vancouver, British Columbia V6T 1W5, Canada 